\title{
TTF-CLASSES OVER PERFECT RINGS
}

\author{
J. S. ALIN and E. P. ARMENDARIZ \\ (Received 17 October 1968; revised 23 April 1969) \\ Communicated by B. Mond
}

For a ring $R$ with unit, let ${ }_{R} \mathscr{M}$ denote the category of unitary left $R$-modules. Following S. E. Dickson [3], a (non-empty) class $\mathscr{P}$ of $R$-modules is a torsion class in ${ }_{R^{\mathscr{M}}} \mathscr{M}$ if $\mathscr{P}$ is closed under factors, extensions, and direct sums. If, in addition, $\mathscr{P}$ is closed under submodules, then $\mathscr{P}$ is said to be hereditary.

An example of a hereditary torsion class is the class $\mathscr{T}=\left\{A \mid \operatorname{Hom}_{R}(A, E(R))\right.$ $=0\}$, where $E(R)$ denotes the injective envelope of $R$. This torsion has been studied in [8], [9], and [11] and, as noted in [11], coincides with usual class of torsion $R$-modules whenever $R$ is a commutative integral domain. In [9], J. P. Jans has established that if $R$ is a right perfect ring in the sense of H. Bass [2], then $\mathscr{T}$ is closed under direct products (i.e., $\mathscr{T}$ is a TTF-class in Jans' terminology). The main purpose of this note is to show that if $R$ is right perfect then every hereditary torsion class is a TTF-class (Corollary 1.6). To further point out the analogy between the class $\mathscr{T}$ and the usual torsion modules, we show that among commutative rings with non-essential singular ideal, integral domains are characterized by the property that $\mathscr{T}$ is the unique maximal element in the lattice of hereditary torsion classes (Theorem 2.2).

\section{$\mathbf{1}$}

Let $\mathscr{C}$ be any class of $R$-modules and let $T(\mathscr{C})$ be the class of all $R$-modules $M$ such that every non-zero homomorphic image of $M$ has a nonzero submodule isomorphic to a member of $\mathscr{C}$. It is readily verified that $T(\mathscr{C})$ is a torsion class which contains $\mathscr{C}$ whenever $\mathscr{C}$ is closed under factors. In addition, if $\mathscr{C}$ is closed under cyclic submodules, $T(\mathscr{C})$ is then hereditary.

Let $\mathscr{S}$ be a representative set of non-isomorphic simple $R$-modules. In [3], Dickson defined the class $\mathscr{D}=T(\mathscr{P})$ (see also [4]). By the previous statements $\mathscr{D}$ is a hereditary torsion class containing $\mathscr{S}$. We shall say a (hereditary) torsion class $\mathscr{P}$ is of simple type if $\mathscr{P}=T(\mathscr{C})$ for some subset $\mathscr{C}$ of $\mathscr{S}$.

Before proceeding, we recall from [3] that if $\mathscr{P}$ is a torsion class, every $R$-module $A$ has a unique maximal $\mathscr{P}$-submodule, $P(A)$ and the factor module $A / P(A)$ has only the zero submodule lying in $P$; i.e., the module $A / P(A)$ is $\mathscr{P}$-torsion-free. 
Proposition 1.1. A hereditary torsion class $\mathscr{P}$ is of simple type if and only if $\mathscr{P} \subseteq \mathscr{D}$.

Proof. If $\mathscr{P} \subseteq \mathscr{D}$, let $\mathscr{C}=\mathscr{P} \cap \mathscr{P}$. Then clearly $T(\mathscr{C}) \subseteq \mathscr{P}$. Let $M \in \mathscr{P}$ and let $N$ be the maximum $T(\mathscr{C})$-submodule of $M$. Then since $M \in \mathscr{D}, M / N=0$ or else $M / N$ has a nonzero simple submodule $S$. If the latter holds, then $S \in \mathscr{C} \subseteq T(\mathscr{C})$ and this contradicts $M / N$ being $T(\mathscr{C})$-torsion-free. Hence $M=$ $N \in T(\mathscr{C})$. The reverse implication is clear.

COROLlaRY 1.2. Every hereditary torsion class in ${ }_{R} \mathscr{M}$ is of simple type if and only if every non-zero $R$-module has a non-zero simple submodule. In particular, if $R$ is right perfect, then all hereditary torsion classes in ${ }_{\mathbf{R}^{\mathscr{H}}} \mathscr{H}$ are of simple type.

We now investigate the internal structure of modules which are torsion with respect to a torsion class of simple type. The method used in the next two lemmas and the resulting theorem is essentially that of Jans [9, Theorem 3.1]. As in [9], a hereditary torsion class closed under direct products will be called a TTF-class.

For an $R$-module $M$ let $s(M)$ denote the socle of $M$. Thus $s(M)=0$ if $M$ has no nonzero simple submodules, or else $s(M)$ is the sum of all nonzero simple submodules of $M$. For each ordinal $\alpha$, define $s^{\alpha}(M)$ as follows:

(1) $s^{0}(M)=0$ and $s^{1}(M)=s(M)$.

(2) If $\alpha=\beta+1$ is not a limit ordinal, define $s^{\alpha}(M)$ by $s^{\alpha}(M) / s^{\beta}(M)=$ $s\left(M / s^{\beta}(M)\right)$.

(3) If $\alpha$ is a limit ordinal, $s^{\alpha}(M)=\bigcup_{\beta<\alpha} s^{\beta}(M)$. The simple modules appearing in $s^{\alpha+1}(M) / s^{\alpha}(M)$ for some ordinal $\alpha$ are called the composition factors of $M$.

LEMMA 1.3. Let $\mathscr{C}$ be a set of simple R-modules and $\mathscr{P}=T(\mathscr{C})$. Then $M \in \mathscr{P}$ if and only if $s^{\alpha}(M)=M$ for some ordinal $\alpha$ and each composition factor of $M$ is isomorphic to a member of $\mathscr{C}$.

Proof. Let $M \in \mathscr{P}$. Then $M / s^{\beta}(M) \neq 0$ implies $s\left(M / s^{\beta}(M)\right) \neq 0$ and hence $s^{\alpha}(M)=M$ for some ordinal $\alpha$. Since $M / s^{\beta}(M) \in \mathscr{P}$ for any ordinal $\beta$, we have $s^{\beta+1}(M) / s^{\beta}(M) \in \mathscr{P}$ since $\mathscr{P}$ is hereditary. Thus each composition factor of $M$ is a member of $\mathscr{P}$ and so is isomorphic to a member of $\mathscr{C}$.

Conversely, suppose $s^{\alpha}(M)=M$ for some $\alpha$ and all composition factors of $M$ are isomorphic to members of $\mathscr{C}$. Let $P(M)$ denote the maximum $P$-torsion submodule of $M$ and suppose $M / P(M) \neq 0$. Choose $\beta$ minimal with respect to $s^{\beta}(M) \nsubseteq P(M)$ and note that $\beta$ is not a limit ordinal. Thus $s^{\beta-1}(M) \subseteq P(M)$ and so there is a non-zero homomorphism $f$ mapping $s^{\beta}(M) / s^{\beta-1}(M)$ into $M / P(M)$. Let $S$ be a simple submodule of $s^{\beta}(M) / s^{\beta-1}(M)$ with $f(S) \neq 0$. Then $f(S) \cong S$ and so $M / P(M)$ has a submodule isomorphic to a member of $\mathscr{C}$, contradicting $P(M / P(M))=0$. Thus $M=P(M)$ and hence $M \in \mathscr{P}$.

Lemma 1.4. Let $M \in \mathscr{D}$ and let $S$ be a simple R-module. Suppose there is an idempotent $e \in R$ such that $e S \neq 0$ and $e S^{\prime}=0$ for all simple modules $S^{\prime}$ not isomorphic to $S$. Then $S$ is a composition factor of $M$ if and only if $e M \neq 0$. 
Proof. If $S$ is a composition factor of $M$ then for some $\alpha, S \subseteq s^{\alpha+1}(M) / s^{\alpha}(M)$, hence $e s^{\alpha+1}(M) \nsubseteq s^{\alpha}(M)$ and so $e M \neq 0$.

On the other hand if $e M \neq 0$ then $e x \neq 0$ for some $x \in M$. Choose $\alpha$ minimal relative to $e x \in s^{\alpha}(M)$. Then $\alpha$ is not a limit ordinal, so $\alpha=\beta+1$ and ex $\notin s^{\beta}(M)$. If $S$ is not isomorphic to a submodule of $s^{\beta+1}(M) / s^{\beta}(M)$, then since $s^{\beta+1}(M) / s^{\beta}(M)$ is a direct sum of simples all non-isomorphic to $S$, we have $e s^{\beta+1}(M) \subseteq s^{\beta}(M)$. But $e^{2} x=e x \in s^{\beta+1}(M)$ and $e x \notin s^{\beta}(M)$. Thus an isomorphic copy of $S$ must occur in $s^{\beta+1}(M) / s^{\beta}(M)$ and hence $S$ is a composition factor of $M$.

THEOREM 1.5. Let $\mathscr{P}$ be a torsion class of simple type, $\left\{S_{\alpha}: \alpha \in A\right\}$ a representative set of non-isomorphic simple $R$-modules, and let $B=\left\{\beta \in A: S_{\beta}\right.$ is $\mathscr{P}$-torsion free $\}$. Suppose for each $\beta \in B$ there is an idempotent $e_{\beta} \in R$ such that $e_{\beta} S_{\beta} \neq 0$ and $e_{\beta} S_{\alpha}=0$ for all $\alpha \in A, \alpha \neq \beta$. Then if $\mathscr{D}$ is a TTF-class, $\mathscr{P}$ is a TTF-class.

Proof. By Lemma 1.3, $M \in \mathscr{P}$ if and only if $s^{\delta}(M)=M$ for some ordinal $\delta$ and all composition factors of $M$ are isomorphic to a member of $\left\{S_{\alpha}: \alpha \in A-B\right\}$. Let $\left\{M_{i}: i \in I\right\} \subseteq P$, and $M=\prod_{i \in I} M_{i}$. Since $D$ is closed under direct products and $\mathscr{P} \subseteq \mathscr{D}, M \in \mathscr{D}$ and so $s^{\delta}(M)=M$ for some ordinal $\delta$. Since each $M_{i} \in \mathscr{P}$, no $S_{\beta}$ is a composition factor of $M_{i}$ for any $\beta \in B$. Thus by Lemma 1.4, $e_{\beta} M_{i}=0$ for all $\beta \in B, i \in I$. Clearly then $e_{\beta} M=0$ for all $\beta \in B$ and so again by Lemma 1.4, each composition factor of $M$ is isomorphic to a member of $\left\{S_{\alpha}: \alpha \in A-B\right\}$. Thus $M \in \mathscr{P}$ and so $\mathscr{P}$ is closed under direct products.

COROLlary 1.6. If $R$ is semi-perfect and $\mathscr{D}$ is a TTF-class, then every hereditary torsion class of simple type is a TTF-class. In particular, if $R$ is right perfect, then every hereditary torsion class in ${ }_{R} \mathscr{M}$ is a TTF-class.

Proof. Let $R$ be semi-perfect with Jacobson radical $N$. Then $R / N$ is Artinian semisimple and so there are only finitely many non-isomorphic simple $R / N$ modules $S_{1}, \cdots, S_{n}$. Choose idempotents $f_{1}, \cdots, f_{n} \in R / N$ satisfying $f_{i} S_{i} \neq 0$ and $f_{i} S_{j}=0$ if $i \neq j$. The idempotents $f_{1}, \cdots, f_{n}$ can be lifted to idempotents $e_{1}, \cdots, e_{n}$ of $R$ [2], and because of the correspondences between simple $R / N$-modules and simple $R$-modules, $S_{1}, \cdots, S_{n}$ are the simple $R$-modules and satisfy $e_{i} S_{i} \neq 0$ and $e_{i} S_{j} \neq 0$ for $i \neq j$. The corollary now follows from the theorem.

It is not in general true that $\mathscr{D}$ closed under direct products implies that all $\mathscr{P}$ of simple type are $T T F$-classes. For let $R_{i}=Z_{p}, i=1,2, \cdots$, where $Z_{p}$ denotes the ring of integers modulo a prime $p$, and let $K=\prod_{i=1}^{\infty} R_{i}$. Now let $R$. be the subring of $K$ generated by $A=\sum_{i=1}^{\infty} R_{i}$ together with the unity of $K$. It is easily checked that every nonzero $R$-module has a non-zero simple submodule. Thus, in view of Corollary 1.2, $\mathscr{D}={ }_{R} \mathscr{M}$ and $\mathscr{D}$ is therefore closed under direct products. Now assume that $\mathscr{P}=T\left(\left\{R_{i}: i=1,2, \cdots,\right\}\right)$ is closed under products. Then $K \in \mathscr{P}$ and thus the simple submodule $R / A$ of $K / A$ belongs to $\mathscr{P}$. Hence $R / A$ is isomorphic to some $R_{i}$, a contradiction to the fact that $A$ is an essential ideal and $R_{i}$ is a direct summand of $R$. Thus $\mathscr{P}$ is not closed under direct products. 
COROLlaRY 1.7. If $\mathscr{D}$ is a TTF-class and $\mathscr{P}$ is a torsion class of simple type such that all $\mathscr{P}$-torsion-free simple modules are projective, then $\mathscr{P}$ is a TTF-class.

Proof. For each projective simple module $S$, there is an idempotent $e \in R$ such that $S \cong K e$. It is easily checked that these idempotents satisfy the condition of Theorem 1.5.

The next statement gives a necessary condition in order that $\mathscr{D}$ be a proper TTF-class. Write

$$
I_{\mathscr{D}}=\cap\{L \mid L \text { is a left ideal of } R \text { and } R / L \in \mathscr{D}\},
$$

and observe that if $\mathscr{D}$ is a $T T F$-class then $R / I_{\mathscr{D}} \in \mathscr{D}$. Thus we have the following

LEMMA 1.8. If $\mathscr{D}$ is a TTF-class then $I_{\mathscr{D}}$ has no maximal submodules.

COROLLARY 1.9. (i) If $I_{\mathscr{D}}$ is a finitely generated $R$-module then $\mathscr{D}$ is a TTF-class if and only if $\mathscr{D}={ }_{R} \mathscr{M}$.

(ii) If $R$ is left noetherian then $\mathscr{D}$ is a TTF-class if and only if $R$ is left artinian.

For an $R$-module $A$ let $Z(A)$ denote its singular submodule [10] and let $\mathscr{Z}=$ $\{A \mid Z(A)=A\}$. In general $\mathscr{Z}$ is not a torsion class, since it is not closed under extensions. However, if $Z(R)=0$ then E. Gentile has shown in [8] that $\mathscr{Z}=\mathscr{T}$; in fact V. Dlab [5] establishes that $\mathscr{Z}=\mathscr{T}$ if and only if $Z(R)=0$ and thus $\mathscr{Z}$ is a torsion class if and only if it coincides with $\mathscr{T}$. Thus for commutative integral domains, $\mathscr{Z}=\mathscr{T}$ is the class of usual torsion modules. The smallest torsion class containing $\mathscr{Z}$ will be denoted by $\mathscr{G}$; i.e., $\mathscr{G}=T(\mathscr{Z})$ (cf. [1] and [6]).

Lemma 2.1. [5]. For any ring $R, \mathscr{T} \subseteq \mathscr{Z}$.

Proof. Suppose $A \in \mathscr{T}$ and $Z(A) \neq A$. If $x \notin Z(A)$ then $(0: x) \cap I=0$ for some left ideal $I \neq 0$ of $R$. Then we have the exact sequence $0 \rightarrow I \rightarrow R x$ with $R x \in \mathscr{T}$. Hence $0 \neq I \in \mathscr{T}$, contrary to $R$ being $\mathscr{T}$-torsion-free.

THEOREM 2.2. Let $R$ be a commutative ring with $G(R) \neq R$. Then $\mathscr{T}$ contains every proper hereditary torsion class if and only if $R$ is an integral domain.

ProOF. If $R$ is an integral domain and $\mathscr{P}$ is any proper hereditary torsion class then $R \notin \mathscr{P}$. Thus if $A \in \mathscr{P}$ and $a \in A$ then $(0: a) \neq 0$ otherwise $R \cong R a \in \mathscr{P}$. Hence every module in $\mathscr{P}$ is a torsion $R$-module and so $\mathscr{P} \subseteq \mathscr{T}$. For the converse we note that since $G(R) \neq R, \mathscr{G}$ is a proper torsion class, and so $\mathscr{Z} \subseteq \mathscr{G} \subseteq \mathscr{T}$. By Lemma $2.1, \mathscr{T} \subseteq \mathscr{Z}$ and hence $\mathscr{T}=\mathscr{Z}$. Thus by Dlab's result [5], $Z(R)=0$ and since $R$ is commutative this means $R$ has no nonzero nilpotent elements. Therefore, fcr any non-zero ideal $I$ of $R$, necessarily 


$$
\{x: x \in I \text { and }(0: x) \supseteq I\}=\{0\} .
$$

Hence the torsion class $\mathscr{P}$ generated by all $R$-modules $M$ such that $I M=0$ satisfies $P(I)=0$. As a consequence $\mathscr{P} \subseteq \mathscr{T} \subseteq \mathscr{Z}$. Hence

$$
R / I=P(R / I)=Z(R / I),
$$

i.e., $I$ is essential in $R$. Thus $R$ is an integral domain as required.

We remark that the condition $G(R) \neq R$ is needed. For let $R$ be a (commutative) local ring $R$ with nilpotent maximal ideal. Evidently $R$ has only two hereditary torsion classes ${ }_{R} \mathscr{M}$ and $0 ; \mathscr{G}={ }_{R} \mathscr{M}$ and $\mathscr{T}=0$.

Finally, we express our appreciation to the referee for his helpful comments concerning the presentation of this paper. He has also called to our attention some recent results of $\mathrm{V}$. Dlab who has obtained (using different methods) a characterization of perfect rings in terms of its torsion classes.

\section{References}

[1] J. S. Alin and S. E. Dickson, 'Goldie's torsion theory and its derived functor', Pacific J. Math. 24 (1968), 195-203.

[2] H. Bass, 'Finitistic dimension and a homological generalization of semi-primary rings', Trans. Amer. Math. Soc. 95 (1960), 466-488.

[3] S. E. Dickson, 'A torsion theory for Abelian categories', Trans. Amer. Math. Soc. 121 (1966), $223-235$.

[4] S. E. Dickson, 'Decomposition of Modules I. Classical rings', Math. Z. 90 (1965), 9-13.

[5] V. Dlab, 'The concept of a torsion module', Amer. Math. Monthly 75 (1968), 973-976.

[6] B. Eckmann and A. Schopf, 'Über injective Modulen', Archiv der Math. 4 (1953), 75-78.

[7] A. W. Goldie, 'Torsion free modules and rings', J. Algebra 1 (1964), 268-287.

[8] E. Gentile, 'Singular submodule and injective hull', Indag. Math. 24 (1962), 426-433.

[9] J. P. Jans, 'Some aspects of torsion', Pacific J. Math. 15 (1965), 1249-1259.

[10] R. E. Johnson, 'The extended centralizer of a ring over a module', Proc. Amer. Math. Soc. 2 (1951), $891-895$.

[11] R. S. Pierce, Modules over Commutative Regular Rings (Memoirs Amer. Math. Soc. 70, Providence, R. I., 1967).

The University of Utah, Salt Lake City, Utah and

The University of Texas, Austin, Texas 